\section{The use of rituximab in newly diagnosed patients with systemic lupus erythematosus: long-term steroid saving capacity and clinical effectiveness}

\author{
Borja Gracia-Tello, ${ }^{1}$ Amara Ezeonyeji, ${ }^{2}$ David Isenberg ${ }^{2}$
}

To cite: Gracia-Tello B, Ezeonyeji $A$, Isenberg $D$. The use of rituximab in newly diagnosed patients with systemic lupus erythematosus: long-term steroid saving capacity and clinical effectiveness. Lupus Science \& Medicine 2017;4: e000182. doi:10.1136/lupus2016-000182

Received 5 August 2016 Revised 31 October 2016 Accepted 23 November 2016

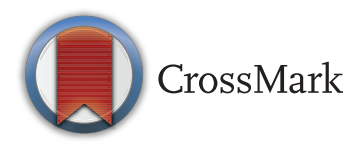

\footnotetext{
${ }^{1}$ Department of Internal Medicine, Lozano Blesa University Hospital, Zaragoza, Spain

${ }^{2}$ Centre for Rheumatology, University College London Hospitals, London, UK
}

\section{Correspondence to} Dr David Isenberg; d.isenberg@ucl.ac.uk

\section{ABSTRACT}

Background: Previous reports indicate that treating patients with lupus (SLE) at or close to the time of diagnosis successfully without using any, or minimal, corticosteroids by using B-cell depletion (BCD) is possible in the short-term. It is not however known whether using BCD is as effective or reduces corticosteroid use in the long-term. We report the long-term (up to 7 years) use of BCD with respect to its steroid-saving capacity and clinical effectiveness in newly diagnosed SLE.

Methods: Sixteen female patients with SLE were treated at, or shortly after diagnosis, with BCD therapy (BCDT) minimising the routine use of oral steroids.

Post-treatment, most patients were given hydroxychloroquine $(n=14)$ and azathioprine $(n=10)$. The British Isles Lupus Assessment Group (BILAG) disease activity index was used for clinical assessment. Serum antidouble-stranded DNA (dsDNA) antibodies, complement (C3), erythrocyte sedimentation rate (ESR), circulating B lymphocytes $\left(\mathrm{CD} 19^{+}\right)$and total inmmunoglobulins were tested every 2-6 months (average of 4.5 years) (SD 2) posttreatment. Disease activity and steroid requirement were compared with three patients with SLE treated conventionally, each matched for ethnicity, sex, age, clinical features, disease duration at diagnosis and follow-up period.

Results: All patients given rituximab achieved BCD. The mean number of flares during follow-up (new BILAG A or B) was 2.63 (SD 3) in the BCDT group and 4 (SD 3.6) in the controls (NS, $p=0.14$ ). Post-BCDT, mean anti-dsDNA antibody level fell from $1114 \mathrm{U} / \mathrm{mL}$ (SD 1699.3) to 194 (SD 346.7) at 18 months $(p=0.043)$, mean serum ESR fell by $>70 \%$ at 6 months maintained during follow-up and serum C3 level normalised in 8 patients. The mean cumulative prednisolone dose at 60 months for the patients who underwent BCDT ( $n=11)$ was $4745.67 \mathrm{mg}$ (SD $6090 \mathrm{mg}$ ) vs $12553.92 \mathrm{mg}$ (SD $12672 \mathrm{mg}$ ) for the controls $(p=0.01)$.

Conclusions: Early treatment of patients with SLE with BCDT is safe, effective and enables a reduction in steroid use.

\section{INTRODUCTION}

SLE is an autoimmune rheumatic disorder associated with a wide spectrum of clinical features. ${ }^{2}$ Randomised controlled trials in SLE are limited, and its treatment usually includes glucocorticosteroids (GG) and hydroxychloroquine for mild to moderate disease and immunosuppressives if severe. ${ }^{34}$

Long-term use of GC and immunosuppressives often leads to side effects that increase morbidity and mortality. ${ }^{5} 6$ Several longitudinal studies, notably those reported by the Systemic Lupus International Collaborating Clinics (SLICC) group have indicated that corticosteroids are the main cause of damage. Thus, the mean SLICC/American College of Rheumatology (ACR) Damage Index (DI) rose from 0.33 at baseline to 1.9 after 15 years of follow-up in an inception cohort. Damage was considered as definitely GC-related in $16 \%$ and $49 \%$ of cases at baseline and last follow-up, respectively. ${ }^{7}$ In another study, the accrual of organ damage correlated with the mean daily prednisone dose, the risk increasing for doses $>6 \mathrm{mg}$ / day. ${ }^{8}$ Every 1-point increase in DI was associated with a 1.32 times more risk to die during follow-up. ${ }^{9}$

To limit GC toxicity, lower oral doses have been successfully used in lupus nephritis (LN) trials, ${ }^{10}$ Other immunosuppressives, such as azathioprine, mycophenolate mofetil (MMF) or cyclophosphamide, are often prescribed in part as steroid-sparing agents. ${ }^{11}$ The availability of biologic agents, notably rituximab (RTX) offers the prospect of an alternative steroid-sparing regime. ${ }^{12}$

B cells play a pivotal role in the pathogenesis of SLE. ${ }^{13}$ Apart from being responsible for autoantibody production, they produce cytokines and chemokines and may act as 
antigen-presenting cells. Anti-B-cell therapy has been widely used to treat SLE. B-cell depletion (BCD) has usually been achieved using RTX, a chimeric anti-CD20 monoclonal antibody often combined with GC and cyclophosphamide. ${ }^{14}$

The efficacy and relative safety of BCD in SLE was suggested by open-label and retrospective studies with good clinical response seen in many patients.

These studies were performed in patients with diverse manifestations notably those for whom conventional treatment had been of limited benefit or caused unacceptable side effects. Following our small study of eight patients followed from diagnosis for 6 months, Condon $e t a l^{15}$ evaluated the effectiveness of treating LN with RTX and MMF at diagnosis. They suggested that oral steroids can be avoided in LN without apparent reduction in efficacy or increase in relapse rates, for up to 3 years.

We now report the long-term (up to 7 years) consequences of BCD therapy (BCDT) in 16 newly diagnosed, mostly non-renal patients with SLE as first-line treatment. We have assessed the long-term GC saving and clinical effectiveness of this approach.

\section{PATIENTS AND METHODS}

Study design and patients

From October 2008 to October 2014, 16 patients with SLE were treated at, or within 3 months of diagnosis, with BCDT aiming to minimise the routine use of oral steroids. Three patients had been given the option to have BCDT or to be treated with conventional treatment including steroids, hydroxychloroquine and immunosuppressives.

We have compared two groups of patients with SLE from University College Hospital. Those patients treated with a defined protocol using BCDT and with at least 1 year follow-up were compared with those from the 'historic cohort' (HC) treated conventionally (usually with steroids). Each of the 16 patients who underwent BCDT was closely matched for ethnicity, sex, age, clinical features, disease duration at diagnosis and length of follow-up with three patients from the HC. Virtually all of the disease controls were treated with conventional therapy during the follow-up period of the BCDTtreated parties.

The groups were followed for an average of 4.56 years (range $1-7$ years).

Patients were excluded if they had previously received previous prolonged treatment notably GC, hydroxychloroquine or immunosuppressive therapy. Two of the patients treated with RTX (patients 6 and 12) had received an intramuscular depomedrone $120 \mathrm{mg}$ injection 2 months before BCDT. Another presented initially elsewhere with active SLE, having been under our care with an undifferentiated autoimmune rheumatic disease. There she was given high-dose oral steroids for 2 weeks before being transferred to our department for
BCD. A third patient had a delayed admission for BCD. Unfortunately, she was admitted as an emergency with active SLE and a concurrent lower respiratory tract infection. She was treated with antibiotics and intravenous methylprednisolone (MP) $750 \mathrm{mg} \times 3$, and was discharged prematurely before she could receive BCD. In total, five patients were treated with oral corticosteroids prior to the infusion of RTX, for a mean of 5.8 weeks (range 1-16 weeks) and a maximum of $730 \mathrm{mg}$ cumulative dose pre-BCDT (patient 2). The treatment was initiated by their general practitioner or the rheumatologist prior to the patient being admitted for BCDT. Eight patients received no GC until they were admitted for BCD.

All patients fulfilled at least four of the revised ACR criteria for the classification of SLE and gave informed consent to treatment before being included in our database. ${ }^{16}$

\section{BCDT protocol}

The standard BCDT treatment protocol used was a combination of $1 \mathrm{~g}$ of RTX on days 1 and 14 and $750 \mathrm{mg}$ of cyclophosphamide (CYC) on day 2. Three patients did not receive CYC with their RTX treatment, and one patient received $500 \mathrm{mg}$ of CYC with the second dose of RTX rather than the first, as she was neutropenic before treatment. One patient received a further $750 \mathrm{mg}$ of CYC with the second RTX dose. MP $100 \mathrm{mg}$ intravenous was administered before each RTX dose.

All patients were followed regularly every 2-6 months. Maintenance therapy was given with the lowest possible prednisone dose, hydroxychloroquine $(\mathrm{n}=14)$ and azathioprine $(\mathrm{n}=11)$ or mycophenolate mofetil (MMF) $(\mathrm{n}=3)$.

When possible, oral steroids were avoided altogether. During follow-up, four patients had to repeat with RTX due to B-cell repopulation, accompanied by a flare, with an average of 21.8 months after the initial dose.

There were no major infections or serious adverse events in patients given BCDT.

\section{$\mathrm{HC}$ treatment}

In 41 of the 48 patients in the $\mathrm{HC}$, prednisolone was prescribed. Hydroxychloroquine was used in 42 as firstline treatment. During follow-up, hydroxychloroquine was stopped in one patient due to gastrointestinal side effects. Azathioprine was used in 21 patients (43.8\%) and MMF in $9(18.8 \%)$. Six patients were given methotrexate and nine $(18.8 \%)$ treated with cyclophosphamide to manage flares.

\section{Variables studied}

BCD was considered to have been achieved once the absolute CD19 count decreased to $<0.005 \times 10^{9} / \mathrm{L}$ after treatment.

Serological markers of disease activity, notably antidouble-stranded DNA (dsDNA antibody titres (by ELISA, normal $<50 \mathrm{IU} / \mathrm{mL}$ ), serum complement (C3) levels (by laser nephelometry-normal $0.90-1.80 \mathrm{~g} / \mathrm{L}$ ), 
erythrocyte sedimentation rate (ESR), circulating B lymphocytes $\left(\mathrm{CD} 19^{+}\right)$and total immunoglobulins were tested every 2-6 months for an average of 4.5 years (SD 2) post-treatment.

The classic British Isles Lupus Assessment Group (BILAG) disease activity index was used for clinical assessment. ${ }^{17}$ The BILAG index was used for both individual organ system assessment and also as a global index A score in any of the eight organ systems=12 points, $\mathrm{B}=5, \mathrm{C}=1$ and $\mathrm{D} / \mathrm{E}=0$. Four of the controls were lost after moving abroad (2.5 years in the control number 3 of patient 1, 1.5 years in the control 3 of patient $6,1.5$ years in control 3 of patient 7 and 1 year in control 3 of patient 12). The SLICC/ACR DI in SLE was calculated in both groups at the end of the follow-up.

The cumulated prednisolone dose was calculated at 1 , 3 and 6 months and subsequently every 6 months during follow-up in both groups.

Clinical and laboratory data were collected prospectively. Treatment efficacy was evaluated on the basis of improvement in both clinical and laboratory features.

Data at 12 months were available for all patients. At 24 months data were available for 14 treated with RTX and 42 of the HC. Data at 3 years were available for 13 patients who underwent BCDT and for 39 controls. Data at 5 years were available for 11 and 33 patients, respectively. The maximum follow-up was of 84 months for two patients treated with RTX and six controls.

\section{Statistical analysis}

Descriptive data were generated. Univariate comparisons between both cohorts were performed using $\chi^{2}$ test or the Fisher's exact test, as appropriate, for qualitative variables, unpaired Student's t-test for quantitative variables with normal distribution and Mann-Whitney $U$ test for quantitative variables with non-normal distribution.

The outcome of both cohorts was compared by several means. Statistical significance was calculated between serological markers of disease activity, immunoglobulins, change in the global BILAG score and cumulative steroid dose at follow-up.

\section{RESULTS}

\section{Patient population}

Sixteen patients were treated with the RTX protocol as the 'first-line' therapy. Follow-up data for a median follow-up of 4.5 years (SD 2; range 1-7 years) were available. The total patient population (BCDT and conventionally treated patients) consisted of 36 Caucasians $(56 \%), \quad 16$ African-Caribbeans $(25 \%)$ and 12 South-Asian and/or Chinese (19\%).

The baseline characteristics of all patients who underwent BCDT are shown in table 1 . The median age of the patients was 34.38 years (range 21-50 years) in the RTX group and 34.67 years (SD 9.2) in the HC $(\mathrm{p}=0.626)$. Median symptom duration before SLE diagnosis was 6 weeks (range 2-384 weeks). One patient (patient 2) had been managed by the dermatologists for 5 years with discoid lupus, Raynaud's phenomenon and arthralgia. During this period, there were no supporting laboratory investigations or other clinical features to make a diagnosis of SLE. However, after referral to rheumatology with worsening symptoms including joint inflammation, a diagnosis of SLE was confirmed.

At diagnosis, all patients who underwent BCDT (B-cell depletion-treated patients (BCDTp)) had joint involvement, 11 had skin manifestations and 3 had serositis. No patients treated with RTX had any gastrointestinal or central nervous system manifestations. Two had renal lupus confirmed on biopsy. In the $\mathrm{HC}$, a similar distribution is evident with $92 \%$ had joint involvement $(\mathrm{p}=0.23)$ almost $60 \%(\mathrm{p}=0.46)$ had skin manifestations, with serositis in $15(\mathrm{p}=0.34)$. Renal lupus was present in nine patients $(p=0.57)$. Two of the HC had autoimmune hepatitis $(\mathrm{p}=0.41)$. A detailed comparison of the BCDT and conventionally treated patients is shown in table 2 . Comparing both groups, no significant differences in initial clinical manifestations distribution were observed.

\section{Serological markers of disease activity}

Circulating B lymphocytes (CD19+)

After 6 months of treatment with RTX, 15 patients maintained circulating B lymphocyte depletion measured by $\mathrm{CD} 19^{+}$with a mean of $0.03 \pm 0.023 \times 10^{9} / \mathrm{L}$. The patient who repopulated at 6 months $\left(\mathrm{CD} 19^{+}\right.$count: $0.121 \times 10^{9} / \mathrm{L}$ ) showed no sign of activity. At the 12 months follow-up $(\mathrm{n}=13)$, all the patients analysed remained B-cell depleted (mean of $0.036 \pm 0.07 \times 10^{9} / \mathrm{L}$ ), although two patients (patient 11 and 14) had required further RTX at 8 months because of a flare, both with successful CD19 ${ }^{+}$B cell depletion.

At 2 years, five of the nine patients remained B-cell depleted with a partial recovery in the $\mathrm{CD} 19^{+}$B-cell number. Only one had needed retreatment with RTX. In one case (patient 3), the depletion persisted until 72 months of follow-up $\left(\mathrm{CD} 19^{+} 0.009 \times 10^{9} / \mathrm{L}\right)$ with no further cycle of RTX needed.

During follow-up, four patients required a new cycle of RTX due to the repopulation of the $\mathrm{CD} 19^{+} \mathrm{B}$ cells and a clinical recurrence. It was successful in three for an average of 15 months. One patient (patient 10), despite maintaining BCD, needed a new cycle of RTX given the persistence of clinical manifestations 5 months after the first treatment.

\section{Inflammatory markers}

At diagnosis, the mean ESR in the BCDTp was 62.13 $\pm 41.5 \mathrm{~mm} /$ hour and $32.89 \pm 29.8 \mathrm{~mm} /$ hour in the HC $(p=0.005)$. At 6 months, the mean ESR fell to 25.94 $\pm 14.8 \mathrm{~mm} /$ hour in the BCDTp and $21.06 \pm 18.5 \mathrm{~mm} /$ hour in the HC. These values were maintained with a steady decline with no significant differences throughout follow-up (table 3 ).

Thirteen out of 16 BCDTp had elevated of anti-dsDNA antibody levels at baseline mean $=1113.87 \pm 1699.4 \mathrm{IU} / \mathrm{mL}$. 
Table 1 The baseline data including organs/systems involved and the treatment given to those patients treated with BCD

\begin{tabular}{|c|c|c|c|c|c|c|c|c|c|}
\hline Patient & $\begin{array}{l}\text { Age of } \\
\text { diagnosis }\end{array}$ & $\begin{array}{l}\text { Follow-up } \\
\text { (years) }\end{array}$ & Ethnicity & $\begin{array}{l}\text { Symptom } \\
\text { duration before } \\
\text { diagnosis } \\
\text { (weeks) }\end{array}$ & $\begin{array}{l}\text { Clinical features and serology at } \\
\text { SLE diagnosis }\end{array}$ & $\begin{array}{l}\text { CYC } \\
\text { post-BCDT }\end{array}$ & $\begin{array}{l}\text { AZA } \\
\text { post-BCDT }\end{array}$ & $\begin{array}{l}\text { MMF } \\
\text { post-BCDT }\end{array}$ & $\begin{array}{l}\text { Additional } \\
\text { RTX }\end{array}$ \\
\hline 1 & 36 & 5 & Caucasian & 5 & $\begin{array}{l}\text { Arthritis, fatigue, ANA, } \uparrow \text { anti-dsDNA, } \\
\text { lymphopenia, } \downarrow \text { C3 }\end{array}$ & Yes & Yes & No & No \\
\hline 2 & 31 & 7 & Caucasian & 384 & $\begin{array}{l}\text { Discoid lupus, malar rash, Raynaud's, } \\
\text { alopecia, pleurisy, } \uparrow \text { anti-dsDNA, } \\
\text { lymphopenia }\end{array}$ & Yes & Yes & No & No \\
\hline 3 & 26 & 6 & $\begin{array}{l}\text { Malaysian/ } \\
\text { Chinese }\end{array}$ & 16 & $\begin{array}{l}\text { Discoid lupus, alopecia, Raynaud's, } \\
\text { arthralgia, ANA, anti-SM, lymphopenia }\end{array}$ & No & Yes & No & No \\
\hline 4 & 39 & 6 & Caucasian & 40 & $\begin{array}{l}\text { Arthritis, serosits, ANA, } \uparrow \text { anti-dsDNA, } \\
\text { lymphopenia, } \uparrow a C L\end{array}$ & Yes & Yes & No & No \\
\hline 5 & 47 & 7 & African-Caribbean & 12 & $\begin{array}{l}\text { Arthritis, rash, alopecia, ANA, } \\
\text { lymphopenia }\end{array}$ & No & No & No & No \\
\hline 6 & 21 & 1 & Chinese & 4 & $\begin{array}{l}\text { Rash, fever, angiooedema, arthritis, } \\
\text { ANA, } \uparrow \text { anti-dsDNA, lymphopenia, } \downarrow \\
\text { C3 }\end{array}$ & Yes & Yes & No & No \\
\hline 7 & 34 & 3 & African-Caribbean & 6 & $\begin{array}{l}\text { Arthritis, rash, alopecia, ANA, } \\
\text { lymphopenia }\end{array}$ & Yes & No & No & Yes \\
\hline 8 & 47 & 6 & African-Caribbean & 6 & $\begin{array}{l}\text { Fever, fatigue, alopecia, rash, arthritis, } \\
\text { serositis, ANA, anti-CCP }\end{array}$ & Yes & Yes & No & No \\
\hline 9 & 44 & 5 & Caucasian & 4 & $\begin{array}{l}\text { Livedo reticularis, serositis, rash, } \\
\text { proteinuria, ANA, } \uparrow \text { anti-dsDNA, } \\
\text { lymphopenia, } \downarrow \text { C3, } \uparrow \text { aCL and LA }\end{array}$ & Yes & Yes & No & No \\
\hline 10 & 29 & 5 & African-Caribbean & 6 & $\begin{array}{l}\text { Arthritis, rash, alopecia, } \uparrow \text { anti-dsDNA, } \\
\downarrow \text { C3, ANA, RNP }\end{array}$ & Yes & Yes & yes & Yes \\
\hline 11 & 50 & 5 & Caucasian & 12 & $\begin{array}{l}\text { Raynaud's, ANA, Ro, arthritis, mouth } \\
\text { ulcers, photosensitivity, rash, Sjögren's } \\
\text { syndrome }\end{array}$ & Yes & Yes & No & Yes \\
\hline 12 & 26 & 1 & Caucasian & 20 & $\begin{array}{l}\text { Arthritis, ANA, p-ANCA, } \uparrow \text { anti-dsDNA, } \\
\text { anti-SM, RNP, } \downarrow \text { C3 }\end{array}$ & No & Yes & No & No \\
\hline 13 & 34 & 2 & Caucasian & 2 & $\begin{array}{l}\text { Arthritis, ANA, rash, Sjögren's } \\
\text { syndrome, } \uparrow \text { anti-dsDNA, } \downarrow \text { C3, renal } \\
\text { failure }\end{array}$ & Yes & No & yes & No \\
\hline 14 & 25 & 6 & Caucasian & 8 & $\begin{array}{l}\text { Arthritis, rash, fever, ANA, } \uparrow \\
\text { anti-dsDNA, anti-SM, RNP, } \downarrow \text { C3 }\end{array}$ & Yes & No & yes & Yes \\
\hline 15 & 28 & 2 & Asian & 4 & $\begin{array}{l}\text { Arthritis, fatigue, fever, } \\
\text { lymphadenopathy, ANA, pleurisy, Ro, } \\
\text { LA, SM and RNP, } \downarrow \text { C3 }\end{array}$ & Yes & Yes & No & No \\
\hline 16 & 32 & 6 & Caucasian & 2 & $\begin{array}{l}\text { Arthritis, headache, Ro, LA, ANA, } \uparrow \\
\text { anti-dsDNA }\end{array}$ & Yes & No & No & No \\
\hline
\end{tabular}


Table 2 Comparison of the BCDT and conventionally treated patients

\begin{tabular}{|c|c|c|c|}
\hline & BCDT group & Control group & \\
\hline \multicolumn{4}{|l|}{ Demographic characteristics } \\
\hline Age (years) & 34.38 & 34.67 & $N D(p=0.626)$ \\
\hline Gender (women/men) & $16 / 0$ & $48 / 0$ & ND \\
\hline \multirow[t]{3}{*}{ Ethnicity } & Caucasian 9 & Caucasians 27 & ND \\
\hline & $\mathrm{A} / \mathrm{C} 4$ & $\mathrm{~A} / \mathrm{C} 12$ & \\
\hline & South-Asian 3 & South-Asian 9 & \\
\hline \multicolumn{4}{|l|}{ Clinical manifestations } \\
\hline Joint involvement & 16 & 44 & $N D(p=0.56)$ \\
\hline Skin manifestations & $11(68.8 \%)$ & $28(58.3 \%)$ & $N D(p=0.46)$ \\
\hline Serositis & $3(18.8 \%)$ & $15(31.3 \%)$ & $N D(p=0.336)$ \\
\hline Pleurisy & $1(6.3 \%)$ & $3(6.3 \%)$ & ND $(p=1.00)$ \\
\hline Renal lupus & $2(12.5 \%)$ & $9(18.8 \%)$ & $N D(p=0.57)$ \\
\hline CNS lupus & 0 & $4(8.3 \%$ & $N D(p=0.233)$ \\
\hline Autoimmune hepatitis & 0 & 2 & $N D(p=0.41)$ \\
\hline BILAG score & 26.68 & 11.08 & $\mathrm{p}=0.005^{\star \star}$ \\
\hline \multicolumn{4}{|l|}{ Serological markers } \\
\hline ESR (mm/hour) & $62.13 \pm 41.5$ & $32.89 \pm 29.8$ & $\mathrm{p}=0.005^{\star \star}$ \\
\hline Complement (g/L) & $0.68 \pm 0.3$ & $1.22 \pm 1.76$ & $\mathrm{p}=0.068^{\star \star}$ \\
\hline Anti-dsDNA (IU/mL) & $1113.87 \pm 1699.4$ & $129 \pm 233$ & $\mathrm{p}=0.001^{\star *}$ \\
\hline $\lg G(g / L)$ & $17.8 \pm 6.09$ & $17.8 \pm 3.4$ & $N D(p=0.98)$ \\
\hline \multicolumn{4}{|l|}{ Treatment } \\
\hline Prednisolone dose at first treatment (mg/day) & 11.5 & $29.12 \pm 35.2$ & $\mathrm{p}=0.02^{\star *}$ \\
\hline
\end{tabular}

Table 3 Serological/blood test results for the patients with BCD and control patients throughout follow-up

\begin{tabular}{|c|c|c|c|c|c|c|c|c|}
\hline \multirow[b]{2}{*}{ Follow-up (months) } & \multicolumn{2}{|c|}{ ESR (mm/hour) } & \multicolumn{2}{|c|}{ Anti-dsDNA (IU/mL) } & \multicolumn{2}{|c|}{$\begin{array}{l}\text { Complement (C3) } \\
\text { (g/L) }\end{array}$} & \multicolumn{2}{|l|}{$\lg G(g / L)$} \\
\hline & BCDT & Control & BCDT & Control & BCDT & Control & BCDT & Control \\
\hline 0 & $62.13 \pm 41.5$ & $32.89 \pm 29.8$ & $1113 \pm 1699$ & $129 \pm 233$ & $0.68 \pm 0.3$ & $1.22 \pm 1.76$ & $17.8 \pm 6.1$ & $17.8 \pm 4.3$ \\
\hline 6 & $25.94 \pm 14.8$ & $21.06 \pm 18.5$ & $551 \pm 984$ & $91 \pm 207$ & $0.87 \pm 0.3$ & $1.37 \pm 2.1$ & $14.1 \pm 5.3$ & $16.8 \pm 6.5$ \\
\hline 12 & $22.06 \pm 16.1$ & $18.54 \pm 4.4$ & $416 \pm 851$ & $110 \pm 262$ & $0.89 \pm 0.2$ & $1.1 \pm 0.6$ & $15.65 \pm 5.6$ & $14.75 \pm 2.8$ \\
\hline 18 & $20.79 \pm 18.8$ & $21.49 \pm 18.9$ & $194 \pm 346.7$ & $105 \pm 273$ & $0.95 \pm 0.3$ & $1.01 \pm 0.3$ & $15.7 \pm 6.6$ & $15.8 \pm 6.9$ \\
\hline 24 & $18 \pm 13.6$ & $22.14 \pm 22.1$ & $158 \pm 208$ & $146 \pm 512$ & $9.98 \pm 0.2$ & $0.98 \pm 0.3$ & $10.34 \pm 5.4$ & $12.17 \pm 3$ \\
\hline 30 & $19.08 \pm 14.5$ & $21.34 \pm 18.9$ & $89.98 \pm 91.3$ & $61 \pm 68$ & $0.99 \pm 0.24$ & $0.99 \pm 0.2$ & $18.6 \pm 5.9$ & $12.17 \pm 3.6$ \\
\hline 36 & $19.42 \pm 15.4$ & $21.58 \pm 25$ & $135 \pm 168$ & $55 \pm 69$ & $1.05 \pm 0.2$ & $1.03 \pm 0.3$ & $14.04 \pm 11$ & $14.96 \pm 11.3$ \\
\hline 42 & $18.33 \pm 14.5$ & $20.82 \pm 22.7$ & $97.5 \pm 112.2$ & $83 \pm 157$ & $1 \pm 0.26$ & $1 \pm 0.25$ & $13.44 \pm 4.9$ & $16.25 \pm 5.8$ \\
\hline 48 & $15.9 \pm 8.2$ & $18.26 \pm 16.3$ & $68.4 \pm 93.4$ & $70 \pm 92$ & $1.12 \pm 0.23$ & $1.03 \pm 0.3$ & $12.82 \pm 7.3$ & $17.47 \pm 8.7$ \\
\hline 54 & $14.1 \pm 8.8$ & $15.36 \pm 13.5$ & $54 \pm 56.2$ & $58 \pm 65$ & $1.12 \pm 0.26$ & $0.99 \pm 0.23$ & $9.7 \pm 6.5$ & $12.17 \pm 2.1$ \\
\hline 60 & $14.09 \pm 10.7$ & $16.18 \pm 13.5$ & $54 \pm 59$ & $76 \pm 81$ & $1.21 \pm 0.4$ & $1.05 \pm 0.3$ & $9.35 \pm 5.2$ & $10.2 \pm 2.2$ \\
\hline
\end{tabular}

In the $\mathrm{HC}$, the mean was $129 \pm 233 \mathrm{IU} / \mathrm{mL}(\mathrm{p}=0.001)$. Six months later, the means were $551.38 \pm 983.9 \mathrm{IU} / \mathrm{mL}$ in BCDTp and $91.37 \pm 206.5 \mathrm{IU} / \mathrm{mL}$ in the HC. This decrease in both groups was maintained throughout the follow-up (table 3), with an average of $53.64 \pm 58.5 \mathrm{IU} / \mathrm{mL}$ in the BCDTp and $76.44 \pm 80.9 \mathrm{IU} / \mathrm{mL}$ in the $\mathrm{HC}$ at 5 years of follow-up.

Only one of the patients in the BCDTp showed increased anti-dsDNA levels during follow-up (patient 6) associated with persistent SLE manifestations despite treatment.

Twelve BCDTp had low complement levels (C3) at baseline. At diagnosis, the mean C3 levels were 0.68 $\pm 0.3 \mathrm{~g} / \mathrm{L}$ in BCDTp and $1.22 \pm 1.76 \mathrm{~g} / \mathrm{L}$ in $\mathrm{HC}$. These values showed a significant increase in the BCDTp reaching $0.869 \pm 0.3 \mathrm{~g} / \mathrm{L}$ at 6 months. During the follow-up (table 3), the BDCTp had a continuous increase of C3 levels until a maximum of $1.21 \pm 0.42 \mathrm{~g} / \mathrm{L}$ at 60 months. In the $\mathrm{HC}$, complement levels were stable throughout the follow-up (table 3 ).

During follow-up, eight patients from BCDTp experienced a progressive increase of C3 levels until normalising for at least 12 consecutive months. The mean time to complement normalisation was 12.75 months.

Finally, the IgG levels showed no significant differences at diagnosis or during the follow-up in both 
groups. At diagnosis, the BCDTp mean was $17.8 \pm 6.09$ and $17.8 \pm 4.3 \mathrm{~g} / \mathrm{L}$ in the HC $(\mathrm{p}=0.98)$. These values were decreasing progressively to a final average of 10.34 $\pm 5.4 \mathrm{~g} / \mathrm{L}$ in the BCDTp and $12.14 \pm 3 \mathrm{~g} / \mathrm{L}$ in the $\mathrm{HC}$ at 2 years of follow-up and $5.2 \pm 9.35$ and $10.2 \pm 2.2 \mathrm{~g} / \mathrm{L}$, respectively at 5 . Overall, IgG levels remained within the normal range throughout the RTX courses. Only one BCDTp (patient 9) showed lower levels of IgG prior to treatment and subsequently during the follow-up. No statistical differences were observed between the two groups.

\section{Activity disease measured by BILAG score}

At diagnosis, mean BILAG global score was 26.68 points (range 12-45) for the BCDTp and 11.08 for the HC. In both groups, the items causing a higher score at diagnosis were related to musculoskeletal manifestations (mean 7.25 in the BCDTp and 2.8 in the HC) followed by mucocutaneous manifestations (mean 2.8 and 1.69, respectively). The mean decrease in global BILAG at 6 months for the BCDTp was -10.8 (range 0-34).

Flares during the follow-up were defined as new BILAG A or B in any of the organs/systems. The global average flares were 2.63 (SD 3.01) in the BCDTp and 4.00 (SD 3.61) in the HC $(p=0.14)$. The BCDT group had an average of 0.58 flares/year and 0.85 flares/year were noted in the control group. This is not statistically significantly different but reveals an encouraging trend. When single organ/system assessment was analysed, no significant differences were found in any of the items.

Sustained improvement following BCDT during the first 6 months of at least two BILAG grades, that is, $\mathrm{A} \rightarrow \mathrm{D}, \mathrm{A} \rightarrow \mathrm{C}, \mathrm{B} \rightarrow \mathrm{D}$ and $\mathrm{B} \rightarrow \mathrm{C}$, in each of the eight organ systems is shown in (table 4).

Manifestations such as rash, arthritis, fatigue, mouth ulcers and pleuritis responded well. At 6 months of initial treatment with RTX, two patients (patient 10 and 13) showed a BILAG worsening; patient 10 had a $C \rightarrow B$ change in a mucocutaneous item and $\mathrm{C} \rightarrow \mathrm{A}$ in musculoskeletal feature. Patient 13 went from $\mathrm{C} \rightarrow \mathrm{B}$ in haematologic involvement. Furthermore, in patients 1, 6 and 9, category B remained unchanged.

At the end of the follow-up, the SLICC/ACR DI scale was calculated for both groups. BCDT group had a mean of 1.06 (SD 1.4) and the HC a mean of 1.35 (SD 1.5) $(\mathrm{p}=0.9)$. In both groups, the item found most frequently was scarring chronic alopecia (3 patients in BCDTp and 11 patients in HC), followed by, in the BCDT group, premature gonadal failure $(n=2)$ and minor tissue loss $(n=2)$. In $\mathrm{HC}$, deforming or erosive arthritis was found in 11 patients, and muscle atrophy or weakness in 4 . In the BCDTp group, eight patients had a final score of 0 , four had a single point, three patients had 3 points and one patient scored 4 points. In HC, 16 patients had a final score of 0,15 patients had 1 point, 9 had 2 points, 3 patients had 3 points, 3 more patients had 4 points, 1 patient had 5 and 1 had 6 points. No differences between groups were found.

\section{Prednisolone treatment}

At first treatment, the mean dose of daily prednisolone was $11.5 \mathrm{mg}$ (range 0-40 mg) in the BCDTp and $29.12 \mathrm{mg}$ (SD $35.2 \mathrm{mg}$ ) in the HC.

At 6 months post-BCDT, the mean cumulative prednisolone dose for the BCDTp was $842.64 \mathrm{mg}$ (SD $854.3 \mathrm{mg}$ ) and $4247.93 \mathrm{mg}$ (SD $613.1 \mathrm{mg}$ ) for the matched controls, with statistical differences between both groups $(p=0.02)$. Subsequently, throughout the follow-up the HC had higher doses of prednisolone with statistically significant differences compared with BCDTp. These values are shown in figure 1. At 5 years of follow-up, BCDTp had accumulated about $1 / 3$ $(37.8 \%)$ of the prednisolone total dose compared with the HC $(\mathrm{p}=0.01)$.

When each patient was assessed, four of the BCDTp did not require maintenance treatment with prednisolone at any time (patients 3, 4, 11 and 12) and one of them only needed oral treatment after 18 months (patient 7).

Initial prednisolone oral dose was $5 \mathrm{mg}$ in two of the patients (8 and 16), $7.5 \mathrm{mg}$ in four (1, 2, 10 and 13), $20 \mathrm{mg}$ in one (patient 15), $25 \mathrm{mg}$ in two (patients 5 and 6) and $40 \mathrm{mg}$ in patient 9.

Patient 14, although initially requiring $30 \mathrm{mg}$ of prednisolone had a lower cumulative dose due to a new treatment with RTX, as discussed previously, allowing subsequent lower doses of steroids. All patients in the BCDTp group had lower cumulated doses than the average of the matched controls except the patient 8 , who remained on $5 \mathrm{mg}$ of prednisolone during the 60 months of follow-up. The cumulative prednisolone dose was 1.5 times higher than the mean of the controls.

\begin{tabular}{|c|c|c|c|c|c|c|c|c|}
\hline BILAG score & Constitutional & Mucocutaneous & Neurological & Musculoskeletal & Cardiorespiratory & Vasculitic & Renal & Haemological \\
\hline Total BILAG A/B scores at baseline & 6 & 7 & 0 & 10 & 0 & 1 & 2 & 3 \\
\hline \multicolumn{9}{|l|}{ Total change at 6 months post-BCDT } \\
\hline$A \rightarrow B$ & 0 & 1 & 0 & 1 & 0 & 0 & 0 & 0 \\
\hline$A \rightarrow C$ & 1 & 2 & 0 & 0 & 0 & 0 & 0 & 0 \\
\hline$A \rightarrow D$ & 0 & 1 & 0 & 1 & 0 & 0 & 0 & 0 \\
\hline$B \rightarrow C$ & 4 & 1 & 0 & 5 & 0 & 0 & 0 & 1 \\
\hline$B \rightarrow D$ & 1 & 2 & 0 & 3 & 0 & 1 & 1 & 0 \\
\hline $\begin{array}{l}\text { BILAG scores A/B remaining } \\
\text { unchanged post-BCDT }\end{array}$ & - & - & - & - & - & - & 1 & 2 \\
\hline
\end{tabular}




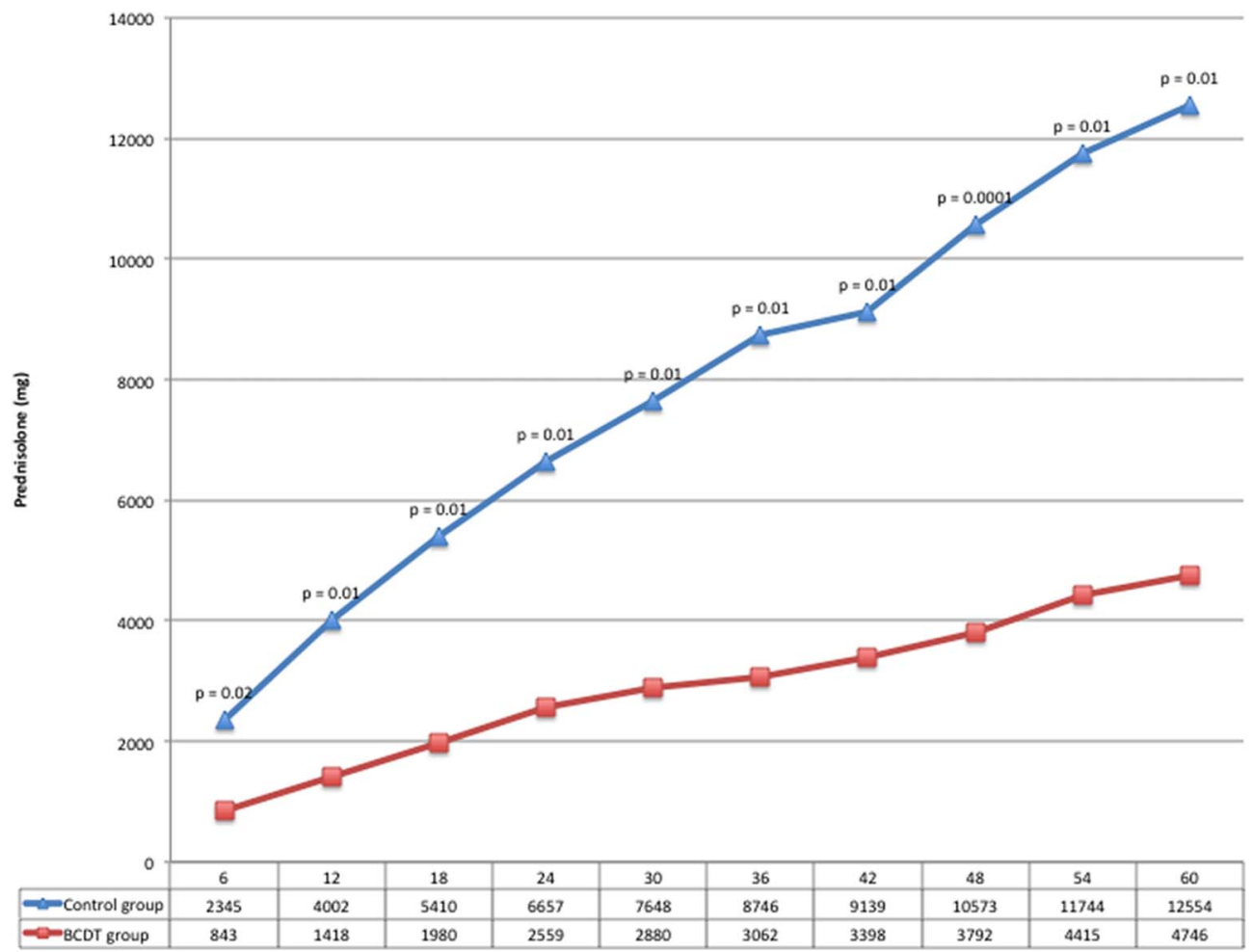

Figure 1 Mean cumulative prednisolone doses for the B-cell depletion treatment (BCDT) and match controls throughout follow-up.

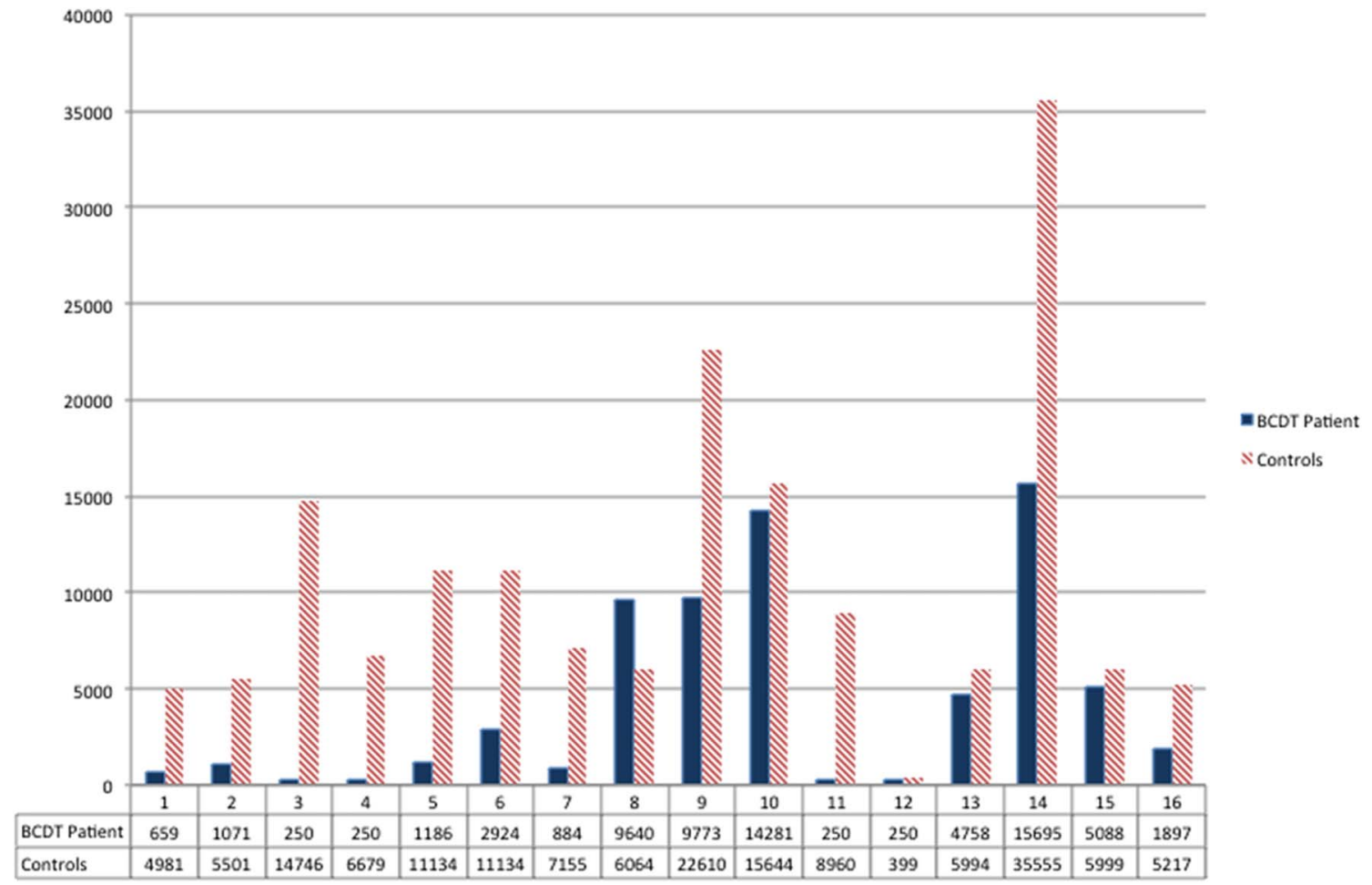

Figure 2 Cumulative prednisolone dose for each of the B-cell depletion patients during follow-up compared with the mean cumulative steroid dose of their respective match controls.

The cumulative prednisolone dose for each of the BCDT patient during the follow-up compared with the mean cumulative steroid dose of their respective matched controls is shown in figure 2.

\section{DISCUSSION}

Survival of patients with SLE has improved dramatically over the past 50 years primarily through the use of GCs, immunosuppressive drugs and the introduction of 
dialysis and renal transplant. ${ }^{13}$ Unfortunately, many of the causes of morbidity in SLE reflect the accumulation of damage occurring as a direct result of the GC therapy. ${ }^{19}$ High-dose GC for long periods has invariably been used to treat severe manifestations of SLE whatever the accompanying immunosuppressive regime. ${ }^{20}$

Although the LUNAR and EXPLORER trials failed to show superiority of RTX-treated patients with SLE over those treated more conventionally, many smaller openlabelled studies have reported a good response to RTX. ${ }^{21-24}$ Ours was the first group to report the use of BCDT early in the treatment of mainly non-renal SLE to help reduce the overall steroid burden. ${ }^{24}$ Initially, we described efficacy at 6-month follow-up in eight patients compared with three carefully matched patients treated with standard therapy.

Neither our report, ${ }^{24}$ the more impressive one of Lightstone $e t a l^{15}$ or the RITUXILUP clinical trial (comparing RTX and MMF with GC and MMF in newly diagnosed LN) can inform the clinician about the long-term efficacy and steroid-saving capacity of BCD used at/close to the time of lupus diagnosis. This 'missing piece in the jigsaw' can only be provided by 'real-life' experience. The data we now provide are very helpful in this regard providing long-term (up to 7 years) follow-up in 16 newly diagnosed patients.

The selection of the controls in this study was dictated by a desire to match them as closely as possible for ethnicity, sex, type of lupus, age at onset, duration of disease at diagnosis and period of follow-up. We did not select them on the basis of matched dsDNA antibody and C3 levels. We were interested to note that the BCDTp had significantly higher dsDNA and lower C3 levels. It is, we suggest, even more impressive therefore that the patients treated with RTX did as well, clinically, over the follow-up period (of several years) compared with the conventionally treated patients and at 5 years of follow-up had accumulated $37.8 \%$ of the prednisolone total dose compared with the controls.

We doubled the number of patients compared with our original study ${ }^{24}$ and extended the follow-up from 6 months to an average of 4.5 years. With a total of 64 patients, 16 early treated with RTX and 48 controls, a cumulative steroid dose reduction was observed at 6 months in the BCDTp, which became even more obvious during the follow-up.

The study shows that the BCDTp had no significant differences in the number of subsequent disease flares. In fact, there was a trend to a reduced number of flares. Treatment with RTX produced a positive clinical response in the patients, measured by the BILAG score, and a progressive decrease in their inflammatory markers including anti-dsDNA antibodies. Finally, the accumulated damage in both groups measured by SLICC/ACR DI showed a trend to a lower score in the BCDTp group, although not statistically significant. These results support the idea that the use of RTX as a first-line treatment has similar efficacy to long-term conventional treatment, but with significantly lower prednisolone dose requirement.

The BCDTp showed no reduction in IgG below the normal range. However, we continue to monitor the IgG levels of all of our patients treated with BCD. These findings were consistent with those of Vital et $a l,{ }^{25}$ who demonstrated that the clinical responses of patients with lupus following RTX are correlated to the B-cell population.

This study has limitations. First, the observational design, with a relatively small number of patients. Second, the heterogeneity in the maintenance treatment and the need to retreat with RTX in four patients during the follow-up. Finally, the use of the classic BILAG underestimates the disease activity of some patients with ophthalmic and gastrointestinal disease as these systems were not included in the classic BILAG scoring.

On balance, we believe that our data support the idea that BCDT may be an effective long-term therapy when used early in the disease. It offers a useful method of reducing the cumulative steroid burden in patients with predominantly non-renal lupus and is at least as effective clinically as the use of more conventional immunosuppressive and corticosteroid drugs.

Contributors DI conceived the project, reviewed the cohort to identify the disease controls, reviewed the data and helped to write the manuscript. BG-T was involved in early discussions of the project, did much of the data collection and writing the first draft of the manuscript and $A E$ was involved in early discussions of the manuscript, some of the data collection and writing the first draft of the manuscript. All authors read and approved the manuscript.

Funding The authors DI and AE are supported by the National Institute for Health Research University College London Hospitals Biomedical Research Centre.

\section{Competing interests None declared}

Ethics approval The use of B-cell depletion in these patients was approved by the hospital's Use of Medicines committee, which considers all aspects of treatment proposed including ethical issues. All patients gave informed consent to the BCD treatment.

Provenance and peer review Not commissioned; externally peer reviewed.

Data sharing statement We have included all the available data in the article as sent to the journal.

Open Access This is an Open Access article distributed in accordance with the Creative Commons Attribution Non Commercial (CC BY-NC 4.0) license, which permits others to distribute, remix, adapt, build upon this work noncommercially, and license their derivative works on different terms, provided the original work is properly cited and the use is non-commercial. See: http:// creativecommons.org/licenses/by-nc/4.0/

\section{REFERENCES}

1. Zen M, Bassi N, Nalotto L, et al. Disease activity patterns in a monocentric cohort of SLE patients: a seven-year follow-up study. Clin Exp Rheumatol 2012;30:856-63.

2. Rahman A, Isenberg DA. Systemic lupus erythematosus. $N$ Engl J Med 2008;358:929-39.

3. Mosca M, Tani C, Aringer M, et al. European League Against Rheumatism recommendations for monitoring patients with systemic lupus erythematosus in clinical practice and in observational studies. Ann Rheum Dis 2010;69:1269-74.

4. Bertsias G, loannidis JP, Boletis J, et al., Task Force of the EULAR Standing Committee for International Clinical Studies Including 
Therapeutics. EULAR recommendations for the management of systemic lupus erythematosus. Report of a Task Force of the EULAR Standing Committee for International Clinical Studies Including Therapeutics. Ann Rheum Dis 2008;67:195-205.

5. Doria A, laccarino L, Ghirardello A, et al. Long-term prognosis and causes of death in systemic lupus erythematosus. Am J Med 2006:119:700-6.

6. Doria A, Gatto M, Zen M, et al. Optimizing outcome in SLE: treating-totarget and definition of treatment goals. Autoimmun Rev 2014;13:770-7.

7. Gladman DD, Urowitz MB, Rahman P, et al. Accrual of organ damage over time in patients with systemic lupus erythematosus. $J$ Rheumatol 2003;30:1955-9.

8. Thamer M, Hernán MA, Zhang $\mathrm{Y}$, et al. Prednisone, lupus activity, and permanent organ damage. J Rheumatol 2009;36:560-4.

9. Chambers SA, Allen E, Rahman A, et al. Damage and mortality in a group of British patients with systemic lupus erythematosus followed up for over 10 years. Rheumatology (Oxford) 2009;48:673-5.

10. Houssiau FA, Vasconcelos C, D'Cruz D, et al. Immunosuppressive therapy in lupus nephritis: the Euro-Lupus Nephritis Trial, a randomized trial of low-dose versus high-dose intravenous cyclophosphamide. Arthritis Rheum 2002;46:2121-31.

11. Borrows R, Loucaidou M, Van Tromp J, et al. Steroid sparing in renal transplantation with tacrolimus and mycophenolate mofetil: three-year results. Transplant Proc 2005;37:1792-4.

12. Pepper R, Griffith M, Kirwan C, et al. Rituximab is an effective treatment for lupus nephritis and allows a reduction in maintenance steroids. Nephrol Dial Transplant 2009;24:3717-23.

13. Leandro MJ, Edwards JC, Cambridge G, et al. An open study of $B$ lymphocyte depletion in systemic lupus erythematosus. Arthritis Rheum 2002;46:2673-7.

14. Gatto M, Kiss E, Naparstek Y, et al. In-/off-label use of biologic therapy in systemic lupus erythematosus. BMC Med 2014;12:30.

15. Condon MB, Ashby D, Pepper RJ, et al. Prospective observational single-centre cohort study to evaluate the effectiveness of treating lupus nephritis with rituximab and mycophenolate mofetil but no oral steroids. Ann Rheum Dis 2013;72:1280-6.

16. Hochberg MC. Updating the American College of Rheumatology revised criteria for the classification of systemic lupus erythematosus. Arthritis Rheum 1997;40:1725.

17. Symmons DPM, Coppock JSA, Bacon PA, et al. Development and assessment of a computerized index of clinical disease activity in systemic lupus erythematosus. Quarterly J Med 1988;69:927-32.

18. Favas C, Isenberg DA. B-cell-depletion therapy in SLE-what are the current prospects for its acceptance? Nat Rev Rheumatol 2009;5:711-16.

19. Urowitz MB, Gladman DD. How to improve morbidity and mortality in systemic lupus erythematosus. Rheumatology (Oxford) 2000;39:238-44.

20. Pagnoux C, Dechartres A, Giraudeau B, et al. Reporting of corticosteroid use in systemic disease trials: evidence from a systematic review of the potential impact on treatment effect. Arthritis Care Res (Hoboken) 2010;62:1002-8.

21. Tanaka $\mathrm{Y}$, Yamamoto $\mathrm{K}$, Takeuchi $\mathrm{T}$, et al. A multicenter phase $\mathrm{I} / \mathrm{II}$ trial of rituximab for refractory systemic lupus erythematosus. Mod Rheumatol 2007:17:191-7.

22. Jónsdóttir T, Gunnarsson I, Mourão AF, et al. Clinical improvements in proliferative vs membranous lupus nephritis following B-cell depletion: pooled data from two cohorts. Rheumatology (Oxford) 2010;49:1502-4.

23. Fernández-Nebro A, de la Fuente JL, Carreño L, et al. Multicenter longitudinal study of B-lymphocyte depletion in refractory systemic lupus erythematosus: the LESIMAB study. Lupus 2012;21:1063-76.

24. Ezeonyeji AN, Isenberg DA. Early treatment with rituximab in newly diagnosed systemic lupus erythematosus patients: a steroid-sparing regimen. Rheumatology (Oxford) 2012;51:476-81.

25. Vital EM, Dass S, Buch MH, et al. B cell biomarkers of rituximab responses in systemic lupus erythematosus. Arthritis Rheum 2011;63:3038-47. 